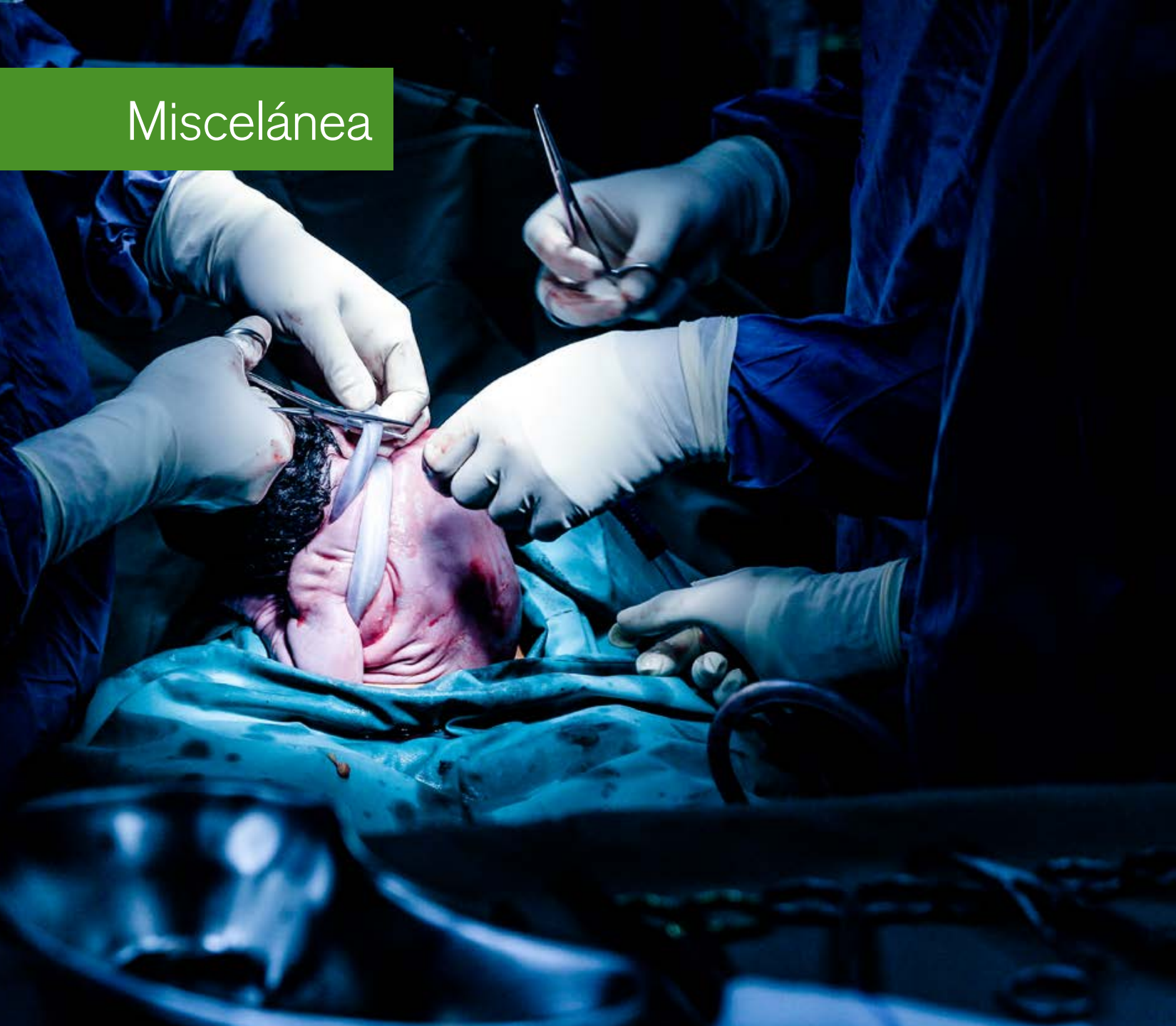

Saúde materna em Moçambique: as taxas de parto cesárea podem ser um indicador de saúde em populações vulneráveis?

Maternal Health in Mozambique: Can Cesarean Section Rates be a Health Indicator in Vulnerable Populations?

B) Autores
Regina Sebastião Inácio

Universidade Católica de Moçambique

E-mail: reginasebas2@gmail.com

(iD) https://orcid.org/0000-0002-0035-6192

\section{Milena Gomes}

Pontifícia Universidade Católica do Paraná

E-mail: gomes.milena@pucpr.edu.br

(iD) https://orcid.org/0000-0001-8221-283X

\section{Carla Corradi-Perini}

Pontifícia Universidade Católica do Paraná

E-mail: carla.corradi@pucpr.br

(D) https://orcid.org/0000-0002-9340-8704 
Resumo

Abstract

Key words
Este estudo tem o objetivo de refletir sobre a saúde materna em Moçambique tendo como ponto central a discussão se as taxas de cesárea nessa população podem ser utilizadas como um indicador de saúde. A justificativa para este estudo parte da premissa que as fragilidades no setor de saúde pública de Moçambique levam ao aumento da realização de cesáreas evitáveis, principalmente, durante a assistência emergencial de mulheres em situação de vulnerabilidade, como exposto na mídia. O material de análise envolve os documentos oficiais do Ministério da Saúde de Moçambique e da Organização Mundial da Saúde. Conclui-se que não existem argumentos para que se tente atingir uma determinada taxa de cesáreas, e sim priorizar o atendimento pré-natal, abrangendo toda a região do país, em especial, para as comunidades mais pobres e que vivem em regiões afastadas dos centros de saúde.

This study seeks to reflect on maternal health in Mozambique, its key point being the discussion of whether the rate of Caesarean sections in this population may be used as a health indicator. The rationale for this study is based on the premise that weaknesses in the public health sector in Mozambique lead to increased Caesarean sections in emergency care for vulnerable women, but these Caesarean sections are preventable, as has been disclosed in the media. The analysed material includes the official documents of the Ministry of Health of Mozambique and the World Health Organization. It concludes that there are no arguments for trying to achieve a certain rate of Caesarean sections, rather what is required is prioritising prenatal care all over the country, especially for poorest communities in regions that are far from health centres.

Cesárea; iniquidade em saúde; cuidado pré-natal.

Cesarean section; health status disparities; prenatal care.

Recibido: 18/07/2020. Aceptado: 27/01/2021 


\section{Introdução}

A saúde materna envolve a saúde da mulher durante a gestação, o parto e o período pós-natal, sendo fundamental para garantia da mesma, o acesso a cuidados de maternidade de qualidade e que respeitem a dignidade das mulheres (OMS, 2020). Apesar dos avanços em saúde nas últimas décadas, no mundo, aproximadamente 295000 mulheres morreram durante o período compreendido entre a gestação e até 42 dias após o parto em 2017, número considerado inaceitavelmente alto pela OMS (2019).

Segundo o Inquérito Demográfico de Saúde (MISAU, INE \& ICFI, 2013), "dar à luz na África Subsariana, incluindo Moçambique, ainda constitui um elevado risco da mortalidade e morbidade associadas com os partos" (p. 122). As principais causas de óbito materno são os abortos clandestinos, a anemia, a eclâmpsia, a hemor-

O processo de gravidez tem exigências específicas de cuidados necessários para a adequada saúde maternoinfantil ao longo de toda a gestação, não se resumindo à atenção apenas no dia do parto ragia, o trabalho de parto obstruído e as infecções puerperais.

A taxa de cesariana é usada como um indicador de disponibilidade e acesso a serviços obstétricos que podem salvar vidas (OMS, 2009). E Moçambique é um dos países que apresenta uma baixa taxa de parto cesárea, principalmente nas áreas rurais e mais pobres (Long, Kempas, Madede, Klemetti, \& Hemminki, 2015). Então, por se tratar de um fenômeno de saúde pública, em que nele devem estar presentes recursos, ações e procedimentos direcionados ao bem-estar físico e mental da paciente, este artigo vem questionar: até que ponto as taxas de parto cesárea podem ser um indicador de saúde em Moçambique?

Ressalta-se que este estudo culmina em um processo reflexivo a partir dos documentos oficiais do Ministério da Saúde de Moçambique, da Organização Mundial da Saúde e motivado especialmente por uma notícia veiculada na mídia relatando a rotina de uma médica que alega que "o número de mães adolescentes que fazem cesarianas está a crescer na Zambézia" (Moçambique) e estima que seja "um parto por dia só de adolescentes" (Mueia, 2018). Muitas adolescentes e mulheres adultas buscam os serviços de partos hospitalares e conforme relato da médica, o parto cesárea de emergência é realizado para não se correr o risco de perder a mulher por causa da ruptura uterina (Mueia, 2018).

A justificativa para este estudo parte da premissa que a assistência emergencial de muIheres para a realização dos partos hospitalares exposta na notícia acima, reflete as fragilidades no setor de saúde pública de Moçambique. E que a interpretação inadequada de eventos como os veiculados na notícia, podem colaborar para a elaboração de novos indicadores que não refletem, de fato, a saúde da população. 0 processo de gravidez tem exigências específicas de cuidados necessários para a adequada saúde materno-infantil ao longo de toda a gestação, não se resumindo à atenção apenas no dia do parto. $A$ prestação de serviços obstétricos básico e de emergência (de qualidade) é um direito de todas as mulheres de Moçambique, atendendo ao princípio da equidade em saúde.

Compreendendo a equidade como a justa e imparcial oportunidade de todas as pessoas em atingir o potencial de sua vida, sem que ninguém seja prejudicado quando isso pode ser evitado (OMS, 1986), este estudo tem o objetivo de refletir sobre a saúde 
materna em Moçambique tendo como ponto central a discussão se a taxa de cesárea nessa população pode ser utilizada como um indicador de saúde.

\section{Parto cesárea versus saúde pública em Moçambique}

Após a aprovação do Plano
Estratégico do Setor da
Saúde pelo governo central,
o Ministério da Saúde de
Moçambique aprovou sua
primeira estratégia nacional
para reduzir a mortalidade
materna em 2001

Apesar de ser um país com elevado nível de pobreza, as políticas de Moçambique expressam que o povo tenha acesso a cuidados de saúde. A missão dos serviços de saúde declarada com o lema "o nosso maior valor é a vida", dá a entender que a saúde e o bem-estar do povo são um direito universal sem distinção (MISAU, 2013).

Um breve histórico dos marcos relacionados à saúde maternoinfantil em Moçambique envolve o lançamento do Programa de Recuperação do Setor da Saúde (HSRP), em 1995. Este programa governamental, com apoio do Banco Mundial, teve como principal objetivo a redução da mortalidade materno-infantil até o ano 2000. Essa meta deveria ser alcançada através da expansão da cobertura de saúde, particularmente da atenção primária à saúde, de $40 \%$ da população estimada para 60\% em 2000 (Lindelow, 2002).

Desde o início deste processo, os indicadores de saúde materno-infantil foram utilizados como os principais parâmetros de desempenho do setor de saúde. Isso criou uma oportunidade muito importante para fortalecer e ampliar as intervenções nessa área. Em 2001, foi lançado o primeiro Plano Estratégico do Setor da Saúde (PESS I: 20012005) e uma das principais intervenções prioritárias foi a saúde materna e infantil. 0 objetivo nesta área foi orientado para a redução da mortalidade materna através da expansão e utilização de informações básicas e abrangentes aos cuidados obstétricos essenciais. As intervenções incluíram medidas preventivas, como planejamento familiar, assistência pré-natal e assistência pós-parto. Após a aprovação do Plano Estratégico do Setor da Saúde pelo governo central, o Ministério da Saúde de Moçambique aprovou sua primeira estratégia nacional para reduzir a mortalidade materna em 2001.

Segundo o Observatório da Equidade de Moçambique (MISAU, 2010), o Ministério de Saúde do Governo de Moçambique, a partir do Plano Estratégico do Setor da Saúde 2007 - 2012 (PESS), reafirma o compromisso com a cobertura universal e com os cuidados de saúde primários. No mesmo relatório, o MISAU (2010) defende como linhas mestras "os cuidados de saúde primários, equidade e melhor qualidade dos cuidados primários" ( $p$. 50), mas reconhece que "mais monitoria e avaliação dos custos e implementação destas disposições são necessárias" (p.50). Adicionalmente, o MISAU destaca que: 
O mais atual Plano Estratégico do Setor da Saúde (PESS) disponível na página do MISAU refere-se ao período de 2014-2019 (MISAU, 2013). Neste PESS, a saúde materna se destaca novamente, sendo o primeiro objetivo listado nos programas de saúde: "Objectivo principal I - Reduzir a morbi-mortalidade através da expansão e melhoria da Qualidade dos Cuidados e Serviços de Saúde Materna" (MISAU, 2013, p. 51). Para atender a esse objetivo foram planejadas estratégias e intervenções no contexto de acesso, qualidade e equidade. Dentre as estratégias e intervenções, incluem-se o fortalecimento do envolvimento comunitário para que a gestante tenha sua primeira consulta pré-natal antes das 16 semanas e para o aconselhamento e mobilização para o parto institucional (incluindo a construção e utilização das casas de espera). Além disso, as estratégias envolvem aumentar a provisão de serviços de saúde materna de qualidade (Atenção Pré-Natal, prevenção da transmissão vertical, parto institucional e consulta pós-parto). Dentre os indicadores, incluem-se: número e porcen-

Os autores alegam que há

a necessidade de continuar

investindo na educação das

mulheres como chave para

promoção da saúde das mães e

crianças tagem de unidades de saúde com Casa de Espera; taxa de cobertura de partos institucionais (por província); porcentagem de partos assistidos em unidades de saúde com cuidados obstétricos de emergência básicos (COEmB) e completos (COEmC) (MISAU, 2013). Cabe ressaltar que a taxa/porcentagem de cesárea não é colocada como um indicador de saúde neste plano. A taxa de cobertura de partos institucionais ou partos assistidos, não refletem, necessariamente, a taxa de cesárea. Entretanto, parece haver o entendimento de que o aumento das taxas de cesárea pode ser interpretado como melhor acesso à saúde.

Os resultados dessas intervenções são evidenciadas no estudo de Chavane e Gonçalves (2019) que analisaram o histórico das iniquidades na saúde materno-infantil em Moçambique nas últimas quatro décadas. Esses autores declaram que há evidências de redução das iniquidades nos indicadores da saúde materno-infantil, embora continue alta quando se considera o nível de escolaridade da mãe. Adicionalmente, os autores alegam que há a necessidade de reduzir as diferenças geográficas no acesso e utilização dos serviços de saúde e a necessidade de continuar investindo na educação das mulheres como chave para promoção da saúde das mães e crianças.

Como descrito na Carta de Ottawa, fruto da I Conferência Internacional de Promoção da Saúde (1986), promoção da saúde é compreendida como "processo de capacitação da comunidade para atuar na melhoria de sua qualidade de vida e saúde, incluindo uma maior participação no controle deste processo". A Carta coloca a saúde como um conceito positivo de bem-estar físico, mental e social. E para atingi-lo, as pessoas devem saber identificar aspirações e satisfazer necessidades. Ou seja, a promoção da saúde envolve um bem-estar global.

As iniquidades no acesso e na utilização dos serviços de saúde contribui de forma negativa para a promoção de saúde. Makanga et al. (2017) exploraram os aspectos geográficos relacionados ao acesso espaço-temporal aos cuidados de saúde. Os autores declaram que muitas gestantes da África Subsaariana tem dificuldades de atendimentos obstétricos, sendo o fator climático, devido a inundações ou altas temperaturas, um obstáculo na procura de atendimento pré-natal e obstétrico para aquelas que 
vivem em regiões mais afastadas dos centros de saúde. Nesse estudo de Makanga et al. (2017), 46\% e 87\% das mulheres grávidas moravam a uma hora do centro de atendimento primário mais próximo, usando os modos de caminhada ou transporte público, respectivamente.

Isto pode justificar as alegações da médica, apresentadas na introdução deste artigo, na notícia veiculada pela Deutsche Welle Notícias, em relação à necessidade de fazer o

A prestação de serviços

obstétricos básicos e de

emergência precisa ser

cuidadosamente projetada para

atender à escassez de serviços

e evitar o desperdício de

recursos de saúde em serviços

desnecessários parto cesárea às mulheres que buscavam o serviço hospitalar de emergência. A médica alegava que esta era uma forma de salvar a vida da mãe e do bebê, principalmente por causa de ruptura uterina (Mueia, 2018). Sendo esta um condição clínica rara em grande parte do mundo, é possível compreender que as barreiras geográficas nesse deslocamento da parturiente até chegar ao serviço de saúde possam levar ao risco de ruptura uterina.

Nesse sentido, a ampliação dos cuidados obstétricos essenciais, colocados como indicadores de saúde em Moçambique (MISAU, 2013), aproximando os serviços das populações mais pobres nas áreas rurais, auxiliariam na redução das taxas de cesárea, principalmente, aquelas evitáveis? Ou seja, há que se interpretar adequadamente os números de partos cesárea. Os indicadores de saúde devem refletir de fato a saúde da população, especificamente, a saúde materna, foco deste estudo.

Long et al. (2015) exploraram as mudanças nas taxas de cesárea em Moçambique em três períodos, de 1995 a 2011. Os autores encontraram que a probabilidade de fazer uma cesárea estava associada a viver em áreas urbanas e na região sul, ter uma educação formal e em melhores condições de moradia, até mesmo ajustando-se à idade e paridade (e períodos de estudo). As taxas de cesarianas variaram de $12,3 \%$ nas mulheres mais ricas em comparação com 1,7\% das mulheres. Na óptica dos autores, deixando de lado o fenômeno pobreza, a situação geográfica e nível de escolaridade é um dos fatores chaves para que a cesárea seja mais realizada nas zonas urbanas. Os autores concluíram que a baixa taxa de cesarianas, particularmente entre grupos socialmente vulneráveis, que são pobres e/ou que vivem em áreas rurais e regiões com poucos recursos, ainda é uma preocupação central em Moçambique. Por outro lado, a taxa de cesarianas pelos grupos mais favorecidos, ricos e vivendo nas grandes cidades, parece estar emergindo. Para finalizar os autores alertam que a prestação de serviços obstétricos básicos e de emergência precisa ser cuidadosamente projetada para atender à escassez de serviços e evitar o desperdício de recursos de saúde em serviços desnecessários (Long et al., 2015).

$\mathrm{Na}$ busca de uma adequada interpretação das informações apresentadas acima, tentou-se explorar o contexto em que esses dados foram produzidos. Interessantemente, foi encontrado que, no ano de 2011 foi introduzido a Taxa Moderadora, para que os pacientes com condições não urgentes (ex. partos eutócicos), tivessem acesso aos cuidados de saúde através de consulta externa (sem serem necessariamente internados). Isso pode ter influenciado o número de pacientes hospitalizados e submetidos à cirurgia (MISAU, 2015). Cabe ressaltar que a gestante que busca o parto assistido, 
pauta por um acompanhamento mais direcionado e cuidadoso com relação ao seu estado e, não uma cesárea, necessariamente. Isto nos faz reforçar o alerta de Long et al. (2015) sobre a necessidade de planejamento adequado da prestação de serviços obstétricos, visando a equidade na assistência.

\section{Para reduzir o número de}

cesáreas evitáveis, a prioridade

deve ser a ampliação do

cuidado pré-natal, que é um dos

principais determinantes da

saúde materna e da sobrevida

do neonato

A declaração vigente da OMS (2015) em relação às taxas de cesáreas auxiliam a responder a questão norteadora deste estudo, a partir de algumas premissas a saber: 1) Os partos cesáreas são eficazes para salvar a vida das parturientes e dos bebês apenas quando tem indicação clínica; 2) A nível populacional, taxas de cesáreas superiores a $10 \%$ não estão associadas com a redução nas taxas de mortalidade materna; 3 ) As cesáreas podem causar complicações, às vezes permanentes, incluindo a morte, especialmente nos locais que carecem de estrutura física e de recursos humanos capacitados para a realização de cirurgias seguras e para tratar as complicações cirúrgicas; 4) Todo o possível deve ser feito para realizar as cesáreas a todas as mulheres que necessitam, ao invés de tentar alcançar uma taxa determinada.

Assim, resgatando o conceito de promoção de saúde com foco na saúde materna, a cesárea constituiria uma alternativa para quando ocorressem complicações durante a gravidez ou parto natural, em que houvesse algum tipo de risco para a mãe, o bebê ou ambos. E para reduzir o número de cesáreas evitáveis, a prioridade deve ser a ampliação do cuidado pré-natal, que é um dos principais determinantes da saúde materna e da sobrevida do neonato. Um rigoroso acompanhamento durante as consultas pré-natais permite a identificação e intervenção precoce de problemas de saúde da mulher e do filho, minimizando danos à saúde materna e infantil (OMS, 2016).

O modelo de assistência pré-natal (APN) de 2016 da OMS, recomenda um mínimo de oito visitas de APN, sendo: um contato no primeiro trimestre, dois no segundo trimestre e cinco no terceiro trimestre. Esse modelo visa proporcionar às gestantes uma assistência centrada na pessoa, que envolve práticas clínicas e eficazes e um atendimento respeitoso e individualizado.

O Ministério da Saúde de Moçambique estabeleceu como indicador a "taxa de cobertura de pelo menos quatro consultas pré-natais" (MISAU, 2013, p.51), estando distante do número recomendado pela OMS (2016). Estratégias para a saúde materna devem ser revistas e efetivamente implantadas, considerando principalmente as populações mais vulneráveis.

\section{Considerações finais}

As políticas públicas em saúde são viáveis quando aplicadas de forma a respeitar o princípio da equidade. Para tanto, é importante que haja responsabilidade e rigor na sua implementação coadjuvado de um efetivo monitoramento (governamental e controle social) a fim de orientar as intervenções e ações por parte das intituições e dos profis- 
sionais de saúde. Além disso, a educação em saúde é uma ferramenta essencial nesse processo de equidade em saúde. O Ministério da Saúde de Moçambique tem estabelecido o compromisso político e constitucional com o acesso universal aos cuidados de saúde a toda sua população. Tem apresentado muitos avanços para a concretização da equidade em saúde, mas, ainda há muito a ser feito em relação à saúde materna.

A partir dos diferentes relatórios oficiais analisados, fica evidente a necessidade de uma ampliação dos serviços de saúde em termos de infraestrutura e de recursos humanos capacitados para uma assistência obstétrica de qualidade, priorizando o atendimento pré-natal, abrangendo toda a região do país, em especial, para as comunidades mais pobres e que vivem em regiões afastadas dos centros de saúde. Destaca-se que muitas gestantes dessas regiões, precisam enfrentar um deslocamento de cerca de uma hora com transporte público ou caminhada para o acesso aos cuidados de saúde. Esse fato ainda se torna mais difícil com a ocorrência de obstá-

A OMS (2015) ressalta que ao invés de se tentar alcançar uma taxa específica de cesárea, os países devem concentrar esforços para garantir que as cesáreas sejam feitas nos casos em que são necessárias culos climáticos como as inundações e as temperaturas elevadas. Além disso, tornam-se importantes as ações intersetoriais, com vistas a mitigar a violação dos direitos sexuais e reprodutivos das mulheres, com um olhar especial às adolescentes.

A resposta à questão que norteou esta pesquisa, "Até que ponto as taxas de parto cesárea podem ser um indicador de saúde em Moçambique?" teve suporte principalmente na Declaração da OMS sobre taxa de cesárea (OMS, 2015). Esse documento invalida a premissa de que as taxas de cesáreas poderiam ser utilizadas como indicador de saúde. A OMS (2015) recomenda que a cesárea, por apresentar riscos à saúde materna, principalmente em locais que carecem de estrutura adequada, somente deve ser realizada quando existe uma indicação clínica. Além disso, ressalta que ao invés de se tentar alcançar uma taxa específica de cesárea, os países devem concentrar esforços para garantir que as cesáreas sejam feitas nos casos em que são necessárias.

Para finalizar, torna-se importante recomendar o seguimento das recomendações da OMS (2018) em relação às intervenções para reduzir as cesáreas desnecessárias. Estas envolvem intervenções dirigidas às mulheres (educação em saúde), aos profissionais de saúde (diretrizes, segunda opinião obrigatória, auditoria e feedback) e às organizações/sistemas de saúde (modelo de assistência colaborativa obstetrícia-obstetra e estratégias financeiras para equalizar as taxas de partos vaginais e cesariana, ambos somente se aplicam em contexto de pesquisas rigorosas). Essas ações, principalmente a educação em saúde, tem o potencial não apenas de redução da taxa de cesáreas desnecessárias, mas a de promoção de saúde para essa população. Além disso, o menor gasto com procedimentos desnecessários possibilita a ampliação dos serviços essenciais de saúde para toda a população. 


\section{Bibliografia}

Carta de Ottawa. In: $1^{a}$ Conferência Internacional sobre Promoção da Saúde. (1986). Ottawa, Canadá. Retrieved from http://bvsms.saude.gov.br/bvs/publicacoes/carta_ottawa.pdf

Chavane, L. A., \& Gonçalves, C. M. de D. (2019). Inequalities in Maternal and Child Health in Mozambique : A Historical Overview. IDS Working paper 526. Brighton.

Lindelow, M. (2002). Health care demand in rural Mozambique: evidence from the 1996 / 97 household survey. FCND discussion paper n.o 126. Washington. Retrieved from http://ageconsearch.umn.edu/bitstream/16420/1/fc020126.pdf

Long, Q., Kempas, T., Madede, T., Klemetti, R., \& Hemminki, E. (2015). Caesarean section rates in Mozambique. BMC Pregnancy and Childbirth, 15(1), 1-9. DOI: https://doi.org/10.1186/s12884-015-0686-X

Makanga, P. T., Schuurman, N., Sacoor, C., Boene, H. E., Vilanculo, F., Vidler, M., Magee, L., von Dadelszen, P., Sevene, E., Munguambe, K., \& Firoz, T. (2017). Seasonal variation in geographical access to maternal health services in regions of southern Mozambique. International Journal of Health Geographics, 1-16. https://doi.org/10.1186/s12942-016-0074-4

Ministério da Saúde (MISAU). (2010). Observatório da Equidade: Avaliação do progresso da equidade na saúde Moçambique. Retrieved from https://www.equinetafrica.org/sites/default/files/uploads/documents/ Moz EW Port Oct2010.pdf

Ministério da Saúde (MISAU). (2013). Plano Estratégico do Sector da Saúde PESS 2014-2019. Maputo. Retrieved from http://www.misau.gov.mz/index.php/planos-estrategicos

Ministério da Saúde (MISAU), Instituto Nacional de Estatística (INE), \& ICF Internacional (ICFI). (2013). Moçambique Inquérito Demográfico e de Saúde 2011. Calverton, Maryland, USA: MISAU, INE e ICFI.

Ministério da Saúde (MISAU). (2015). Análise da mortalidade nacional - Moçambique 2009-2013. Maputo. Retrieved from https://www.moasis.org.mz/wp-content/uploads/2015/08/Publication_Relatorio-analisecompleta-SIS-ROH-2009-2013_Final.pdf

Mueia, M. (2018). Moçambique: Cresce o número de cesarianas em adolescentes na Zambézia. Deutsche Welle Notícias. Retrieved from https://www.dw.com/pt-002/moçambique-cresce-o-número-de-cesarianas-em-adolescentes-na-zambézia/a-42735849

Organização Mundial da Saúde (OMS). (1986). Social justice and Equity in Health, a Report of a WHO Meeting. Copenhagen.

Organização Mundial da Saúde (OMS). (2009). Monitoring Emergency Obstetric Care. Genebra: WHO Press.

Organização Mundial da Saúde (OMS). (2015). Declaração da OMS sobre taxas de cesáreas. Genebra. Retrieved from https://apps.who.int/iris/bitstream/handle/10665/161442/WHO_RHR_15.02_por.pdf?sequence=3

Organização Mundial da Saúde(OMS). (2016). WHO recommendations on antenatal care for a positive pregnancy experience. Retrieved from https://apps.who.int/iris/bitstream/handle/10665/250796/9789241549912eng.pdf;jsessionid=9EFD5473EC466AA3447CE04330127D00? sequence=1

Organização Mundial da Saúde (OMS). (2018). WHO recommendations: non-clinical interventions to reduce unnecessary caesarean sections. Genebra. Retrieved from https://www.who.int/reproductivehealth/publications/non-clinical-interventions-to-reduce-cs/en/ 
Organização Mundial da Saúde (OMS). (2019). Maternal mortality : level and trends 2000 to 2017. Sexual and Reproductive Health. Retrieved from https://www.who.int/reproductivehealth/publications/maternalmortality-2000-2017/en/

Organização Mundial da Saúde (OMS). (2020). Maternal Health. Retrieved July 14, 2020, from https://www. who.int/health-topics/maternal-health\#tab=tab_1 